\title{
RelB deficiency causes combined immunodeficiency
}

\author{
Daniele Merico ${ }^{a}$, Nigel Sharfe ${ }^{b}$, Pingzhao Hu${ }^{a, c}$, Jo-Anne Herbrick ${ }^{a}$, and Chaim M. \\ Roifman ${ }^{\mathrm{b}}$
}

\begin{abstract}
Background: Combined immunodeficiency (CID) presents in infancy with severe microbial infections due to either the depletion or dysfunction of lymphocytes. Several mutated genes have been implicated in causing this condition. These encoded proteins are involved in gene recombination, signal transduction from receptors to transcription factors, or they are critical for lymphocyte development. There remain $20 \%-30 \%$ of patients with similar phenotypes but with no known genetic aberration. The objective of this study was to define the molecular basis of CID in a group of patients.

Genotyping was performed using linkage panel chips, and the results were analyzed for parametric linkage. Whole genome sequencing was also performed. In vitro mitogen stimulation, flow cytometry, real time PCR, Western blotting, and cytokine ELISA were used to assess immunological status and signal transduction pathways.

We identified a homozygous mutation in the gene for the NFKB transcription factor RelB in 3 patients who suffered repeated infection despite the presence of circulating $\mathrm{T}$ and $\mathrm{B}$ cells. This mutation introduces a premature stop, resulting in an ablation of RelB expression. Evaluation of patient immune systems revealed reduced response to mitogens and an inability to maintain an adequate antibody response to immunizations.
\end{abstract}

Lack of RelB expression results in a clinical presentation of CID.

Statement of novelty: We describe RelB deficiency for the first time.

\section{Introduction}

The nuclear factor kappa-B (NFkB) family of transcription factors consists of 5 members: RelA (p65), RelB, Rel (c-Rel), and 2 precursor proteins NFkB1 (p105) and NFkB2 (p100), which are processed to p50 and p52, respectively (Sun 2011; Millet et al. 2013). Two major pathways of NFkB activation are recognized: the classical, mediated by RelA (and c-Rel); and the less understood alternative, mediated by RelB. Infectious agents (through toll receptors), inflammatory cytokines, and lymphocyte antigen receptor activation all activate NFkB activity (Sun 2011; Millet et al. 2013).

All the NFkB proteins contain a Rel homology domain necessary for DNA binding. However, transactivation and nuclear localization domains are separated between proteins, requiring the formation of dimers to obtain an active complex with nuclear targeting ability. Classical pathway activation is regulated by the binding of inhibitory IкB proteins to Rel-A/p105 complexes, sequestering them in the cytoplasm (Sun

${ }^{a}$ Centre for Applied Genomics, Department of Pediatric Laboratory Medicine and the Hospital for Sick Children, University of Toronto, Toronto, ON, Canada; ${ }^{\mathrm{b}}$ Immunogenomic Laboratory, Division of Immunology/Allergy, Department of Pediatrics, The Canadian Centre for Primary Immunodeficiency, the Hospital for Sick Children, Toronto, ON, Canada; ${ }^{c}$ Department of Biochemistry and Medical Genetics, George and Fay Yee Centre for Healthcare Innovation, University of Manitoba, Winnipeg, MB, Canada

*Correspondence: Chaim M. Roifman/chaim.roifman@sickkids.ca Nigel Sharfe and Daniele Merico contributed equally to this paper.

Submitted 27 February 2015 Accepted 11 March 2015

Available online 11 March 2015 
2011; Millet et al. 2013). Phosphorylation of ІкB proteins by IKK kinases initiates IKB degradation, freeing the NFkB complexes to then enter the nucleus. In contrast, RelB activity is suppressed by the binding of NFKB2 p100; alternative pathway activation requiring inducible proteolytic processing of p100 to its p52 form (initiated by NIK and IKK $\alpha$ ) to permit RelB nuclear entry (Sun 2011; Millet et al. 2013).

Although RelA is activated by a large number of receptors (e.g., TNFR, TLR4, TCR), RelB activity is observed downstream from only a handful (BAFF-R, CD40, TWEAK, Lymphotoxin- $\beta-\mathrm{R}$ ), despite its wide expression. Furthermore, although these receptors utilize the alternative pathway, simultaneous activation of the classical pathway is observed. In addition to its receptor activated functions, the presence of RelB is proposed to be necessary for the regulation of other family members (Oeckinghaus et al. 2011; Sun 2011; Millet et al. 2013). RelB knockout mice show that the protein plays an important role in the development of secondary lymphoid tissue (Burkly et al. 1995; Weih et al. 1995). Furthermore, most $\mathrm{RelB}^{-/-}$mice die young, owing to overwhelming multi-organ inflammatory processes stemming from aberrant T-cell cytokine secretion and (or) antigen recognition.

Human primary immune deficiencies have previously been shown to arise from mutations in NFKB Essential Modulator (NEMO, which is also known as inhibitor of nuclear factor kappa-B kinase subunit gamma $(I K K \gamma)), I \kappa B \alpha$, and Inhibitor of NFK-B kinase subunit beta (IKKR, which is also known as IKK2) (Doffinger et al. 2001; Courtois et al. 2003; Janssen et al. 2004; Orange et al. 2004; Lopez-Granados et al. 2008; Picard et al. 2011; Pannicke et al. 2013; Schimke et al. 2013). We show here that RelB deficiency in humans is associated with a combined immunodeficiency (CID).

\section{Methods}

Patient data were compiled prospectively and retrospectively from medical records and were entered into the Canadian Centre for Primary Immunodeficiency Registry and tissue bank, which was approved by the SickKids Research Ethics board (protocol no. 1000005598). This includes consent and assent from patients and parents for genetic analysis and the collection of tissue including thymus. Patients were treated primarily at The Hospital for Sick Children, Toronto, Ontario.

\section{Linkage analysis}

MERLIN (Abecasis et al. 2002) was used for parametric linkage analysis. Minor allele frequency was established based in HapMap CEU (North Americans and Northern and Western European descent) and TSI (Italians from Tuscany) samples. The parametric logarithm base 10 of odds (LOD) score was calculated for a 1 centiMorgan (cM) grid along the chromosomes at disease allele frequency of $1 \%$.

\section{Whole genome sequencing}

The whole genome of 1 patient (patient 2) was sequenced using the Complete Genomics (Mountain View, California, USA) platform.

\section{T- and B-cell proliferative responses}

Lymphocyte proliferative responses to mitogens (including phytohemagglutinin (PHA) and anti-CD3 antibodies) and to a panel of recall antigens (including candida, tetanus, herpes zoster, and cytomegalovirus) were determined by thymidine incorporation at day 3 or day 6. All assays were performed in triplicate and were compared with simultaneously stimulated random normal controls.

\section{Immunoglobulin and specific antibody determinations}

Serum concentrations of immunoglobulins were measured by nephelometry. Serum IgE concentration was measured by radioimmunoassay with the IgE PRIST kit (Pharmacia Diagnostics, Quebec, Canada). Levels of serum antibodies to tetanus were measured by ELISA and polio antibody titers were determined by complement fixation.

\section{Real-time polymerase chain reaction (RT-PCR)}

RT-PCR was performed on an ABI 7500 real-time

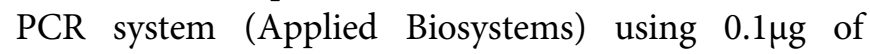
cDNA with the SYBR select Master Mix (Life Technologies). We used the following primers:

GAPDH-F: GAAACTGTGGCGTGATGGC, GAPDH-R: CACCACTGACACGTTGGCAG, RELB-F: CCCTACGAGGACCTGGAGAT, and RELB-R: GCAGGAAGTGGTCCAGGAT. 


\section{Western blotting}

Whole-cell lysates were prepared in a RIPA buffer and analyzed by Western blotting. Anti-RelA, c-Rel,

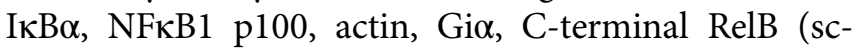
226), and N-terminal RelB antibody (sc-38007) were purchased from Santa Cruz Biotechnology Inc. (Dallas, Texas, USA). Anti N-terminal RelB (04-1077) was also purchased from EMD Millipore (Billerica, Massachusetts, USA). Where necessary, expression levels were quantified by densitometry scanning and normalized by comparison with matching control protein levels. All blots were repeated at least twice.

\section{Results}

\section{Case reports}

Patient 1 was born after cesarean section at 41 weeks to consanguineous parents of Irish descent. At the age of 4 months, patient 1 began experiencing persistent cough and had 3 episodes of pneumonia, all of which required hospitalization. In addition, he suffered multiple episodes of otitis media and required tympanostomy tubes.

Patient 2, the younger brother of patient 1, was born at term by scheduled cesarean section. From the age of 1 month he had a chronic cough and repeated upper respiratory infections. At the age of 13 months, he was admitted for ecthyma gangrenosum which was confirmed by skin biopsy (Figure 1). He has suffered multiple episodes of lobar pneumonia that required antibiotic treatment and hospitalization, as well as failure to thrive. He was noticed to have intermittent swelling of his knees with limping.

Patient 3, a first cousin of patients 1 and 2, was born at term. Immediately after birth he developed pneumothorax and was kept in hospital for 7 days. Growth and development have been slow, and he remains small at less than 10th percentile for height and weight. He has a chronic cough and has had reactive airway disease since infancy. He suffered multiple episodes of otitis media as well as pneumonia and 1 episode of urinary tract infection. He had visible tonsillar tissue as well as palpable lymph nodes.

\section{Evaluation of the immune system}

All patients displayed normal numbers of circulating white blood cells upon presentation. Immunophenotyping revealed that circulating lymphocyte numbers were normal to elevated in all 3 patients. (Table 1). Although the
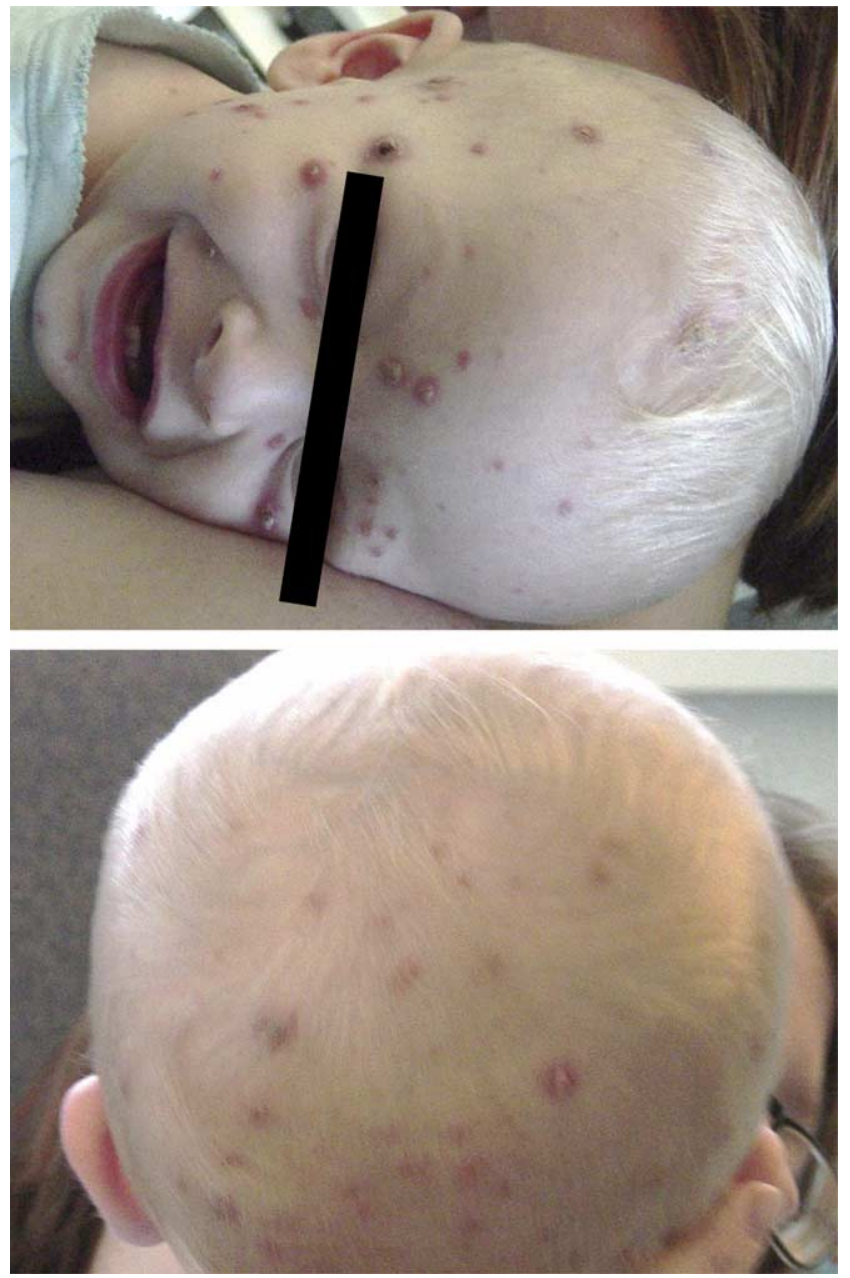

Figure 1: Skin manifestations in patient 2; skin lesions are consistent with ecthyma gangrenosum.

number of circulating $\mathrm{CD}^{+} \mathrm{T}$ cells was within normal range for age, $\mathrm{CD} 4^{+} \mathrm{T}$ cells were somewhat increased, resulting in aberrant CD4:CD8 ratios of 4-10:1 (controls being 1.8-2.5:1). CD20 positive B-cell numbers were also significantly elevated, whereas CD56 positive natural killer cells were normal.

Despite the number of circulating $\mathrm{T}$ cells, in-vitro responses to PHA were less than $50 \%$ of normal controls and in-vitro responses to antigens including candida, zoster, simplex, and cytomegalovirus were completely flat (not shown). Total serum immunoglobulin levels were normal, but the ability to produce specific antibodies in vivo appeared to be significantly impaired (Table 1).

\section{Linkage analysis}

Four family members, corresponding to the 2 male affected siblings and the parents, were analyzed using 
Table 1: Immunological Evaluation

\begin{tabular}{|c|c|c|c|c|}
\hline Markers (cells/ $\mu \mathrm{L})$ & Patient 1 & Patient 2 & Patient 3 & Normal range \\
\hline CD3 & 4195 & 1464 & 3523 & $2300-5400$ \\
\hline CD4 & 3460 & 1122 & 2668 & $700-2200$ \\
\hline CD8 & 522 & 288 & 856 & $490-1300$ \\
\hline CD56 & 1075 & 600 & 868 & $390-1400$ \\
\hline CD19 & 5135 & 3804 & 1912 & $130-720$ \\
\hline \multicolumn{5}{|c|}{ Mitogenic response ( $\%$ control) } \\
\hline $\mathrm{PHA}$ & 36 & 22 & 25 & $>50 \%$ \\
\hline Anti-CD3 & 24 & 2 & 10 & $>50 \%$ \\
\hline \multicolumn{5}{|l|}{ Immunoglobulins (g/L) } \\
\hline $\lg G$ & 5.4 & 14.2 & 8.5 & $3.9-14$ \\
\hline $\lg A$ & 0.4 & 0.5 & 0.2 & $0.4-3.7$ \\
\hline $\lg M$ & 0.5 & 1.2 & 0.9 & $0.5-3.1$ \\
\hline Anti-tetanus (IU/mL) & $<0.01$ & $<0.01$ & $<0.01$ & $>0.01$ \\
\hline
\end{tabular}

Illumina linkage panel chip (5913 single nucleotide polymorphisms (SNPs)). All of the 4 samples had a call rate greater than $98.9 \%$. The self-reported gender and pedigree structure were confirmed by genotype data analysis using PLINK (Purcell et al. 2007).

MERLIN (Abecasis et al. 2002) was used for parametric linkage analysis. The minor allele frequency of the 4135 common SNPs present in both HapMap III and the Illumina linkage panel were estimated based on HapMap $\mathrm{CEU}^{1}$ samples. Disease was assumed to be autosomal recessive with full penetrance as: 0.0, 0.1, and 1.0 (no mutated allele, 1 mutated allele, and 2 mutated alleles, respectively).

The parametric LOD score was calculated for a $1 \mathrm{cM}$ grid along the chromosomes at disease allele frequency of $1 \%$. Four chromosome regions were identified with a maximum LOD score larger than 2 (hg19 genomic coordinates) (Figure 2):

Chromosome 3 (i): start = rs11720298, $70.9405 \mathrm{cM}, 51581$ 509 (hg19); end = rs1472653, 80.094 cM, 59585592 (hg19);

Chromosome 3 (ii): start $=$ rs1317244, $120.54 \mathrm{cM}, 112053$ 565 (hg19); end = rs1052620, 144.177 cM, 136574521 (hg19)

Chromosome 18: start = rs736839, $70.0197 \mathrm{cM}, 46528065$ (hg19); end = rs1539964, 97.8493 cM, 67 625,285 (hg19)

Chromosome 19: start $=$ rs1055099, $69.3765 \mathrm{cM}, 43979$ 589 (hg19); end = rs1017379, 91.09 cM, 52457306 (hg19)

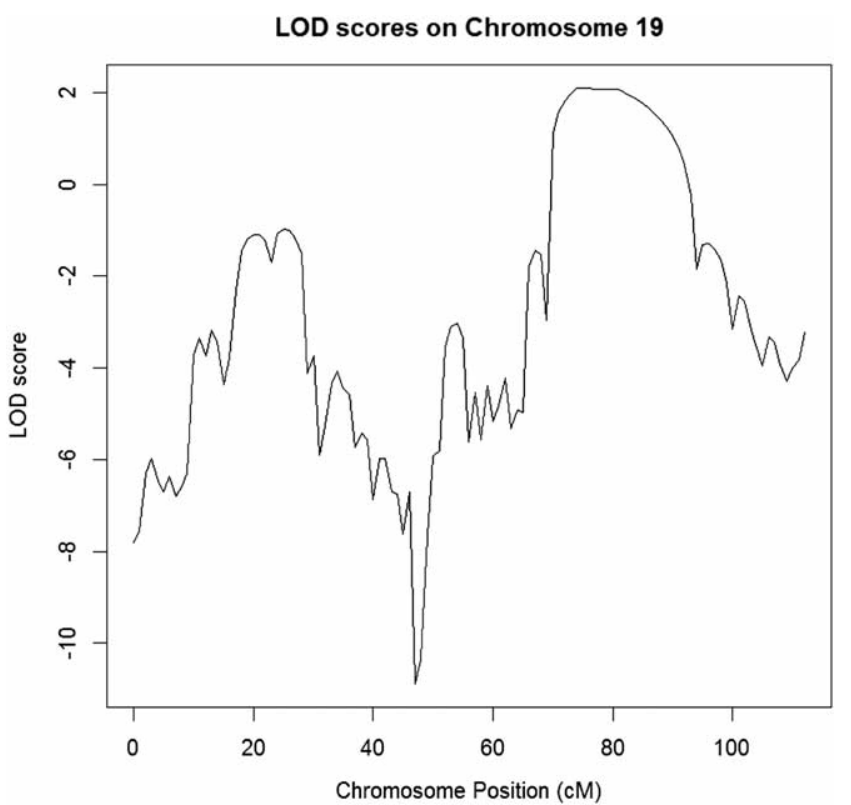

Figure 2: Logarithm base 10 of odds (LOD) scores for chromosome 19. The parametric LOD score for a $1 \mathrm{cM}$ grid along the chromosomes at disease allele frequency of $1 \%$ was calculated. Four chromosome regions with maximum LOD score larger than 2 were found. Chromosome 19 is shown here, for which the LOD $>2$ region starts at rs1055099 (69.3765 cM, hg19: 43,979,589) and ends at rs1017379 (91.09 cM, hg19: 52,457,306).

\section{Whole genome sequencing}

The whole genome of patient 2 was sequenced using the Complete Genomics (Mountain View, Calif.) platform. The concentration of genomic DNA sample

${ }^{1} \mathrm{CEU}$ are Utah residents with Northern and Western European ancestry from the CEPH (Centre d'Etude du Polymorphisme Humain), which is the organization that collected the information. 
was measured with picogreen, and approximately $10 \mathrm{mg}$ of DNA was submitted to Complete Genomics for whole genome sequencing. Complete Genomics employs high-density DNA nanoarrays that are concatemers of mate pair reads, each approximately $500 \mathrm{bp}$ long. Base identification was performed using a nonsequential, unchained read technology known as combinatorial probe-anchor ligation (cPAL). Each mate pair includes $35 \mathrm{nt}$ of genomic DNA sequence as well as adaptor sequences required for $\mathrm{CPAL}$ sequencing; the average mate gap length is $300 \mathrm{bp}$.

The resulting whole genome sequence had a fully called genome fraction (i.e., excluding sites with nocalls) of $96.7 \%$ and a fully called exome fraction of $98.3 \%$. The genome fraction at weighted coverage $\geq 10$ was $97.7 \%$ and the corresponding exome fraction was $98.8 \%$; in particular, $>60 \%$ of the genome was covered at $>40 \times$ - summary statistics indicative of a high-quality sequencing run.

\section{Disease variant identification}

The search for the disease variant(s) was initiated by applying the following filters on single nucleotide variants, small indels, and small block substitutions: (i) restrict to the 4 linkage peaks; (ii) restrict to highquality variants; we selected variants with the varQuality field of the Complete Genomics MasterVarBeta file equal to VQHIGH (corresponding to VAF, i.e., variable allele fraction model, quality score for both alleles equal or greater than $20 \mathrm{db}$ for homozygous calls, and equal or greater than 40 for other calls); and (iii) restrict to variants annotated as "coding" or "splicing" by either Complete Genomics' annotations or in-house annotations based on Annovar (Wang et al. 2010).

This procedure resulted in 50-500 variants per peak. We further restricted to homozygous variants, assuming a recessive model, and excluded synonymous variants. Variants were manually reviewed, taking into account: (i) variant frequency in reference databases (1000 Genomes, NHLBI-ESP, Complete Genomics reference panel) (Abecasis et al. 2010; Tennessen et al. 2012) and presence in dbSNP137; (ii) residue-level conservation (PhyloP score) and local sequence conservation (multi-species alignments as provided by the UCSC browser); (iii) variant effect on coding sequence (e.g., missense, stop-gain, splicing); and (iv) gene function, provided by Gene Ontology (Ashburner et al. 2002), and associated phenotypes, provided by HPO/OMIM (OMIM descriptions as processed into a controlled

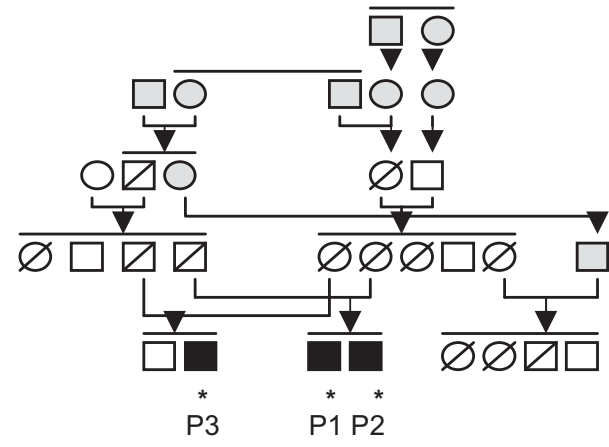

Figure 3: RelB Y397stop segregates with disease. (Affected individuals (patients P1P3) are indicated by asterisks. Black, homozygous; diagonal hash, heterozygous; grey, unknown genotype; square, male; circle, female.)

vocabulary by the Human Phenotype Ontology) and MPO/MGI (Mammalian Phenotype Ontology annotations for mouse genes provided by Mouse Genome Informatics) (Smith et al. 2005; Robinson et al. 2008).

We identified a homozygous stop-gain in the gene RelB (chr19: 45,535,991). Both alleles had a high-quality score (variable allele fraction model: 111 and $981 \mathrm{db}$; equal allele fraction model: 50 and $52 \mathrm{db}$ ) and the variant was novel according to all reference databases (dbSNP, 1000 Genomes, NHLBI-ESP, Complete Genomics reference panel). Inspection of the locus in UCSC did not reveal any anomaly. In addition, NHLBI-ESP has not reported any stop-gain or splicing variant for RelB in over 5000 subjects, suggesting that the gene is heavily under negative selection against loss of function variants; it is specifically interesting that no loss of function altering the final exons were found either.

Subsequently, the stop-gain mutation was confirmed by Sanger sequencing and found to segregate with disease following the analysis of 21 family members (Figure 3). All 3 affected children were homozygous for the RelB mutation, all 4 parents were heterozygous, and unaffected siblings and close relatives were either heterozygous or carrying 2 wild-type alleles.

\section{Quantification of RelB mRNA and protein levels}

Relative levels of RelB mRNA were assessed by RTPCR analysis in 2 patients to determine whether the stop-gain mutation affected RelB mRNA transcription. 
Table 2: mRNA levels of RelB in patients.

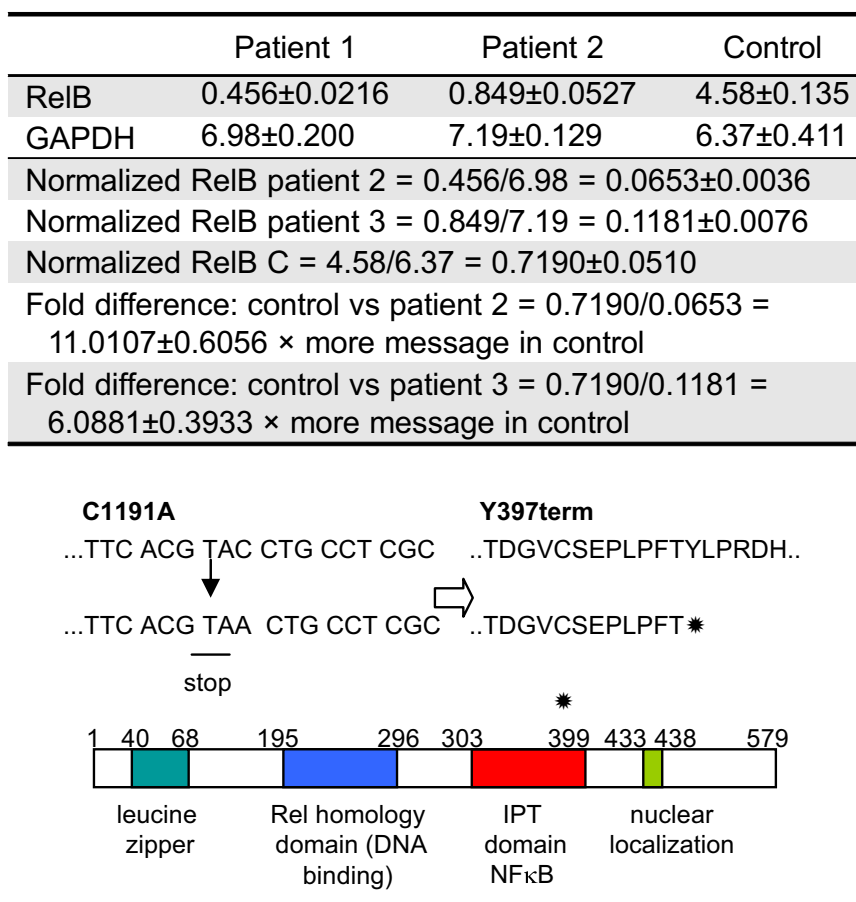

Figure 4: RelB indicating mutation and consequences for protein translation.

Total RNA was isolated from Epstein-Barr virus (EBV) transformed B-cell lines (normal control, patient 2, and patient 3) and reverse transcription carried out with SuperScript III RT and Oligo(dT)12-18 primer. Primer pairs were checked for specificity by standard PCR. Relative quantification was performed using the standard curve method. The normal control EBV cDNA was used as the calibrator and GAPDH as an endogenous control. Results were determined as the mean and standard deviation for 5 replicate samples within a single experiment. In both patients examined, RelB mRNA levels were found to be lower than normal control (Table 2).

The mutation identified in $\operatorname{RelB}$ created a premature stop codon that blocked translation of the last 3 coding exons, shortening the protein by approximately onethird (terminating at amino acid 396) and potentially creating a protein devoid of transcription activating properties, owing to the deletion of its C-terminal nuclear localization sequence (Figure 4). Western blotting with antibodies against the RelB C-terminus, predicted to be deleted by the mutation, revealed no detectable RelB expression in the peripheral blood lymphocyte (PBL) of patient 1 and patient 3 (Figure 5). Furthermore, antibodies against the retained RelB $\mathrm{N}$-terminal region also failed to detect any RelB protein in patient lysates. Enrichment by immunoprecipitation with anti- $\mathrm{N}$ terminus antibody and subsequent Western blotting similarly failed to detect any potential RelB fragment that would be coded by amino acids 1-396 if translated (predicted fragment, $43 \mathrm{kDa}$; wild type, $62 \mathrm{kDa}$ ) (Figure 5B).

In contrast to RelB, all other components of the $\mathrm{NF \kappa B}$ alternative and classical signaling pathways that appeared to be normally expressed were examined in patient EBV B cells and primary fibroblasts (Figure 6). Thus, the premature stop codon appears to effectively ablate RelB expression in these patients, resulting in a "knockout" phenotype.

\section{Discussion}

We describe here a novel type of CID associated with a mutation in the RelB gene. Patients presented with repeated infections and failure to thrive, hallmarks of severe combined immunodeficiency (SCID). As these patients have normal to increased (yet dysfunctional) numbers of circulating $T$ cells, they would be classified as leaky SCIDS or a CID. Occasionally, these types of patients may have autoimmune manifestations such as Omenn syndrome or colitis, yet management and outcome are similar to SCID in most cases (Roifman et al. 2012).

Patient consanguinity facilitated linkage analysis, several clear peaks with significant LOD scores were identified that ultimately helped pinpoint a homozygous mutations in RelB on chromosome 19. This premature stop codon mutation leads to decreased levels of RelB mRNA, presumably due to message instability. Furthermore, the RelB protein was undetectable in patient lymphocytes, suggesting that any potential fragment that may be translated is also unstable and subsequently degraded.

$\mathrm{RelB}^{-1-}$ knockout mice die young of overwhelming inflammation in multiple organs owing to a T-cell dependent infiltration of tissues by myeloid lineage cells (although the exact mechanism is unclear) (Weih et al. 1995, 1996). In contrast, humans with RelB deficiency do not develop this T-cell driven disease, but rather suffer repeated infections and persist with chronic lung and gastro-intestinal symptoms. Patients with defects of $N E M O$ or $I \kappa B \alpha$ in the related NFKB classical pathway may present with anhidrotic ectodermal dysplasia; however, this was not seen in our patients. RelB deficient patients did not display atypical mycobacterial 
A

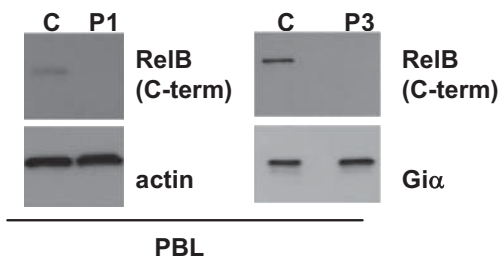

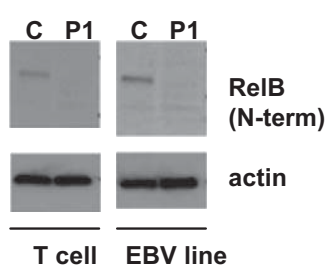

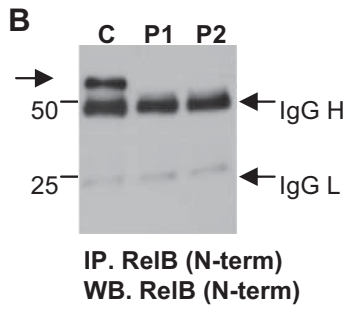

Figure 5: RelB expression in patient PBL and EBV B-cell lines by blotting with antibodies to the RelB C-terminal (predicted to be deleted) and anti-RelB N-terminal (retained) (A). To enrich the potential RelB fragment, immunoprecipitation was also performed from $10^{7} \mathrm{EBV} B$ cells with antibodies to the RelB $\mathrm{N}$ terminus and blotted with anti-N-terminal, but no remnant fragment was detected (B).

A
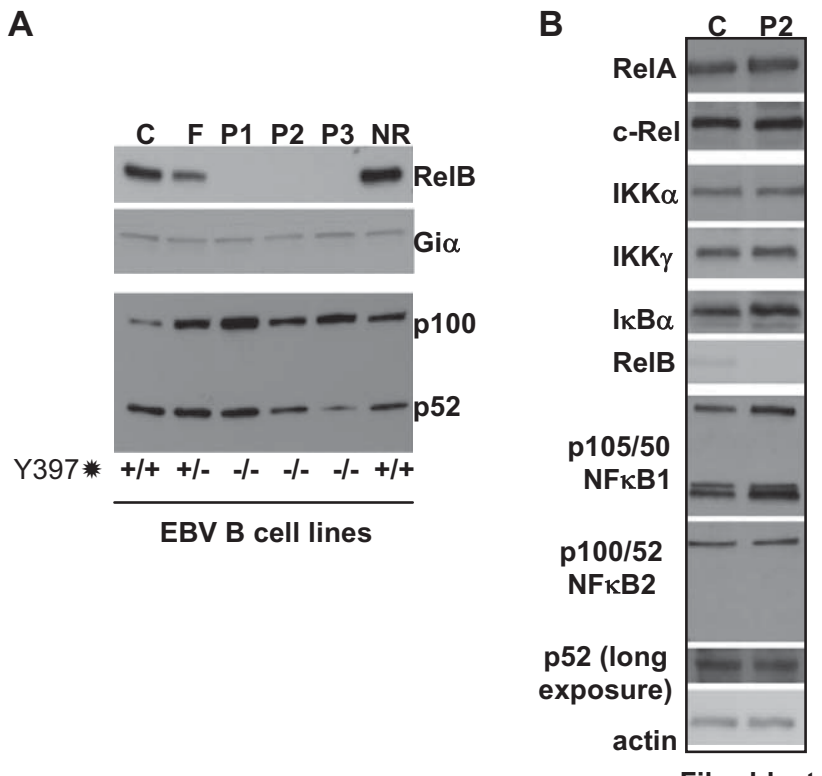

Figure 6: Other members of the NFkB signaling pathways, both classical and alternative, were not significantly altered where assessed, in patient EBV B-cell lines and fibroblasts (A and B). (Patients 1-3, P1, P2, P3; father of P3, F; wildtype/wild-type relative,NR; and unrelated controls,C.) Gix or actin is shown as the loading control where appropriate. All experiments were repeated at least twice.

susceptibility either, as observed in classical pathway deficiencies.

Invariably, all 3 patients had failure to thrive, a typical manifestation of combined immunodeficiency. Evaluation of the immune system revealed dysfunctional $\mathrm{T}$ cells that were unresponsive to mitogens or antigens in-vitro, and humoral immunity was also severely affected, with a failure to respond to childhood vaccines. Ultimately, symptoms were severe enough to warrant treatment with hematopoietic stem cell therapy.

The fact that RelB, a member of the alternative pathway of NFKB activation, appears so critical for the function of the immune system is surprising. RelB activity has been reported in signaling pathways downstream from only a few receptors: BAFF-R, CD40, TWEAK, and Lymphotoxin- $\beta-R$, suggesting that its deletion might have caused minimal phenotype. It is likely that the immune defects apparent in RelB-deficient humans are due to a wider effect of RelB on the classical NFkB pathway (involving RelA and c-Rel) through crossregulation of activation and expression in addition to the function of RelB within the alternate pathway.

\section{ACKNOWLEDGEMENTS}

This work was supported by the Program for Immunogenomics and the Canadian Centre for Primary Immunodeficiency, the Jeffrey Modell Foundation, and Immunodeficiency Canada. The Centre for Applied Genomics at SickKids was supported by Genome Canada through the Ontario Genomics Institute, Canada Foundation for Innovation, and the Ontario Ministry of Research and Innovation.

\section{REFERENCES}

Abecasis, G.R., Altshuler, D., Auton, A., Brooks, L.D., Durbin, R.M., Gibbs, R.A., Hurles, M.E., and McVean, G.A. 2010. A map of human genome variation from population-scale sequencing. 1000 Genomes Project Consortium. Nature. 467(7319):1061-1073. PMID: 20981092. doi: 10.1038/nature09534.

Abecasis, G.R., Cherny, S.S., Cookson, W.O., and Cardon, L.R. 2002. Merlin-rapid analysis of dense genetic maps using sparse gene flow trees. Nat. Genet. 30(1):97-101.

Ashburner, M., Ball, C.A., Blake, J.A., Botstein, D., Butler, H., Cherry, J.M., Davis, A.P., Dolinski, K., Dwight, S.S., Eppig, J.T., Harris, M.A., Hill, D.P., Issel-Tarver, L., Kasarskis, A., Lewis, S., Matese, J.C., Richardson, J.E., Ringwald, M., Rubin, G.M., and Sherlock, G. 2002. Gene ontology: tool for the unification of 
biology. The Gene Ontology Consortium. Nat. Genet. 25(1):25-29. PMID: 10802651. doi: 10.1038/75556.

Burkly, L., Hession, C., Ogata, L., Reilly, C., Marconi, L. A., Olson, D., Tizard, R., Cate, R., and Lo, D. 1995. Expression of relB is required for the development of thymic medulla and dendritic cells. Nature. 373(6514):531-536. PMID: 7845467. doi: 10.1038/ $373531 \mathrm{a} 0$.

Courtois, G., Smahi, A., Reichenbach, J., Doffinger, R., Cancrini, C., Bonnet, M., Puel, A., Chable-Bessia, C., Yamaoka, S., Feinberg, J., Dupuis-Girod, S., Bodemer, C., Livadiotti, S., Novelli, F., Rossi, P., Fischer, A., Israel, A., Munnich, A., Le Deist, F., and Casanova, J.L. 2003. A hypermorphic IkappaBalpha mutation is associated with autosomal dominant anhidrotic ectodermal dysplasia and $\mathrm{T}$ cell immunodeficiency. J. Clin. Invest. 112(7):1108-1115.

Doffinger, R., Smahi, A., Bessia, C., Geissmann, F., Feinberg, J., Durandy, A., Bodemer, C., Kenwrick, S., Dupuis-Girod, S., Blanche, S., Wood, P., Rabia, S.H., Headon, D.J., Overbeek, P.A., Le Deist, F., Holland, S.M., Belani, K., Kumararatne, D.S., Fischer, A., Shapiro, R., Conley, M.E., Reimund, E., Kalhoff, H., Abinun, M., Munnich, A., Israel, A., Courtois, G., and Casanova, J.L. 2001. X-linked anhidrotic ectodermal dysplasia with immunodeficiency is caused by impaired NF-kappaB signaling. Nat. Genet. 27 (3):277-285. PMID: 11242109. doi: 10.1038/85837.

Janssen, R., van Wengen, A., Hoeve, M.A., ten Dam, M., van der Burg, M., van Dongen, J., van de Vosse, E., van Tol, M., Bredius, R., Ottenhoff, T.H., Weemaes, C., van Dissel, J.T., and Lankester, A. 2004. The same IkappaBalpha mutation in two related individuals leads to completely different clinical syndromes. J. Exp. Med. 200(5):559-568. PMID: 15337789. doi: 10.1084/jem.20040773.

Lopez-Granados, E., Keenan, J.E., Kinney, M.C., Leo, H., Jain, N., Ma, C.A., Quinones, R., Gelfand, E.W., and Jain, A. 2008. A novel mutation in NFKBIA/ IKBA results in a degradation-resistant $\mathrm{N}$-truncated protein and is associated with ectodermal dysplasia with immunodeficiency. Hum. Mutat. 29(6):861868. PMID: 18412279. doi: 10.1002/humu.20740.

Millet, P., McCall, C., and Yoza, B. 2013. RelB: an outlier in leukocyte biology. J. Leukoc. Biol. 94(5):941-951. PMID: 23922380. doi: 10.1189/jlb.0513305.

Oeckinghaus, A., Hayden, M.S., and Ghosh, S. 2011. Crosstalk in NF-kappaB signaling pathways. Nat. Immunol. 12(8):695-708. PMID: 21772278. doi: 10.1038/ni.2065.

Orange, J.S., Jain, A., Ballas, Z.K., Schneider, L.C., Geha, R.S., and Bonilla, F.A. 2004. The presentation and natural history of immunodeficiency caused by nuclear factor kappaB essential modulator mutation. J. Allergy. Clin. Immunol. 113(4):725-733. PMID: 15100680. doi: 10.1016/j.jaci.2004.01.762.

Pannicke, U., Baumann, B., Fuchs, S., Henneke, P., Rensing-Ehl, A., Rizzi, M., Janda, A., Hese, K., Schlesier, M., Holzmann, K., Borte, S., Laux, C., Rump, E. M., Rosenberg, A., Zelinski, T., Schrezenmeier, H., Wirth, T., Ehl, S., Schroeder, M.L., and Schwarz, K. 2013. Deficiency of innate and acquired immunity caused by an IKBKB mutation. N. Engl. J. Med. 369(26): 2504-2514. PMID: 24369075. doi: 10.1056/NEJMoa 1309199.

Picard, C., Casanova, J.L., and Puel, A. 2011. Infectious diseases in patients with IRAK-4, MyD88, NEMO, or IkappaBalpha deficiency. Clin. Microbiol. Rev. 24 (3):490-497. PMID: 21734245. doi: 10.1128/ CMR.00001-11.

Purcell, S., Neale, B., Todd-Brown, K., Thomas, L., Ferreira, M.A., Bender, D., Maller, J., Sklar, P., de Bakker, P.I., Daly, M.J., and Sham, P.C. 2007. PLINK: a tool set for whole-genome association and populationbased linkage analyses. Am. J. Hum. Genet. 81 (3):559-575. PMID: 17701901. doi: 10.1086/519795.

Robinson, P.N., Kohler, S., Bauer, S., Seelow, D., Horn, D., and Mundlos, S. 2008. The human phenotype ontology: a tool for annotating and analyzing human hereditary disease. Am. J. Hum. Genet. 83(5):610615. doi: 10.1016/j.ajhg.2008.09.017.

Roifman, C.M., Somech, R., Kavadas, F., Pires, L., Nahum, A., Dalal, I., and Grunebaum, E. 2012. Defining combined immunodeficiency. J. Allergy. Clin. Immunol. 130(1):177-183. PMID: 22664165. doi: 10.1016/j.jaci.2012.04.029.

Schimke, L.F., Rieber, N., Rylaarsdam, S., Cabral-Marques, O., Hubbard, N., Puel, A., Kallmann, L., Sombke, S.A., Notheis, G., Schwarz, H.P., Kammer, B., Hokfelt, T., Repp, R., Picard, C., Casanova, J.L., Belohradsky, B.H., Albert, M.H., Ochs, H.D., Renner, E.D., and Torgerson, T.R. 2013. A novel gain-of-function IKBA mutation underlies ectodermal dysplasia with immunodeficiency and polyendocrinopathy. J. Clin. Immunol. 33(6):1088-1099. PMID: 23708964. doi: 10.1007/s10875-013-9906-1.

Smith, C.L., Goldsmith, C.A., and Eppig, J.T. 2005. The mammalian phenotype ontology as a tool for annotating, analyzing and comparing phenotypic information. Genome. Biol. 6(1):R7. PMID: 15642099. doi: 10.1186/gb-2004-6-1-r7.

Sun, S.C. 2011. Non-canonical NF-kappaB signaling pathway. Cell. Res. 21(1):71-85. doi: 10.1038/cr.2010. 177. 
Tennessen, J.A., Bigham, A.W., O'Connor, T.D., Fu, W., Kenny, E.E., Gravel, S., McGee, S., Do, R., Liu, X., Jun, G., Kang, H.M., Jordan, D., Leal, S.M., Gabriel, S., Rieder, M.J., Abecasis, G., Altshuler, D., Nickerson, D.A., Boerwinkle, E., Sunyaev, S., Bustamante, C.D., Bamshad, M.J., and Akey, J.M. 2012. Evolution and functional impact of rare coding variation from deep sequencing of human exomes. Science. 337(6090): 64-69. doi: 10.1126/science.1219240.

Wang, K., Li, M., and Hakonarson, H. 2010. ANNOVAR: functional annotation of genetic variants from highthroughput sequencing data. Nucleic. Acids. Res. 38 (16):e164. doi: 10.1093/nar/gkq603.
Weih, F., Carrasco, D., Durham, S.K., Barton, D.S., Rizzo, C.A., Ryseck, R.P., Lira, S.A., and Bravo, R. 1995. Multiorgan inflammation and hematopoietic abnormalities in mice with a targeted disruption of RelB, a member of the NF-kappa B/Rel family. Cell. 80(2):331-340. PMID: 7834753. doi: 10.1016/00928674(95)90416-6.

Weih, F., Durham, S.K., Barton, D.S., Sha, W.C., Baltimore, D., and Bravo, R. 1996. Both multiorgan inflammation and myeloid hyperplasia in RelB-deficient mice are $\mathrm{T}$ cell dependent. J. Immunol. 157(9): 3974-3979. PMID: 8892630. 